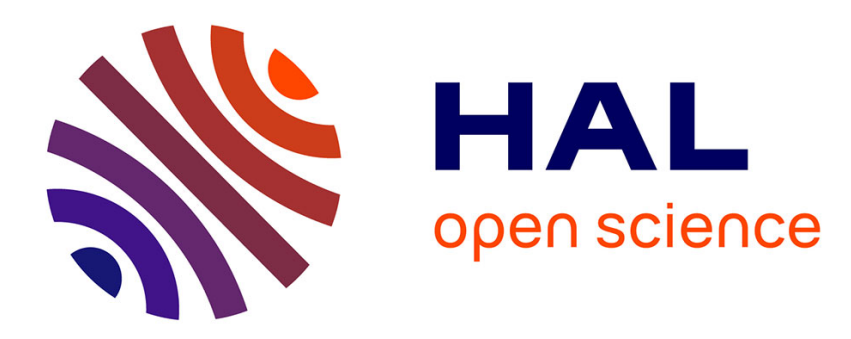

\title{
Using User Contextual Profile for Recommendation in Collaborations
}

\author{
Siying Li, Marie-Hélène Abel, Elsa Negre
}

\section{To cite this version:}

Siying Li, Marie-Hélène Abel, Elsa Negre. Using User Contextual Profile for Recommendation in Collaborations. Research and Innovation Forum 2019, Apr 2019, Rome, Italy. pp.199-209, 10.1007/9783-030-30809-4_19. hal-03218720

\section{HAL Id: hal-03218720 \\ https://hal.science/hal-03218720}

Submitted on 5 Jul 2021

HAL is a multi-disciplinary open access archive for the deposit and dissemination of scientific research documents, whether they are published or not. The documents may come from teaching and research institutions in France or abroad, or from public or private research centers.
L'archive ouverte pluridisciplinaire HAL, est destinée au dépôt et à la diffusion de documents scientifiques de niveau recherche, publiés ou non, émanant des établissements d'enseignement et de recherche français ou étrangers, des laboratoires publics ou privés. 


\title{
Using User Contextual Profile for Recommendation in Collaborations
}

\author{
Siying $\mathrm{Li}^{1}$, Marie-Hélène Abel ${ }^{1}$ and Elsa Negre ${ }^{2}$ \\ ${ }^{1}$ Sorbonne universités, Université de technologie de Compiègne, CNRS UMR 7253, \\ HEUDIASYC, 60203 Compiègne, France \\ \{siying.li, marie-helene.abel\}@utc.fr \\ ${ }^{2}$ Paris-Dauphine University, PSL Research University, CNRS UMR 7243, LAMSADE, 75016 \\ Paris, France \\ elsa.negreddauphine.fr
}

\begin{abstract}
Nowadays, many digital technologies are developed to support collaboration and facilitate its efficiency. When using them, users will leave contextual information explicitly and implicitly, which could contribute to identifying users' situations and thus enabling systems to generate corresponding recommendations. In the framework of collaborations, we are interested in considering user context with user contextual profile to suggest appropriate collaborators. In this article, we present the user contextual profile that we established and how it can be used to generate recommendations for collaborations in digital environments.
\end{abstract}

Keywords: Context-aware recommendations, Contextual profile, Collaboration.

\section{Introduction}

Nowadays, more and more people consider collaborations as an effective way to work [3], particularly with the rapid development of digital technologies, such as blogs [5], wiki [5] and digital ecosystems [6]. These bring great convenience to collaborations from the term of tools [4] and environments [3]. However, at the same time, these technologies also pose problems in acquiring relevant information about collaborators, which are caused by information overload [8]. Thus, finding appropriate collaborators remains an urgent problem to be solved [12]. In order to tackle it, many researchers have dedicated great effort to recommend collaborators [8, 9, 12]. As part of our research, we focus on recommending collaborators to a user of a digital collaborative environment. We aim at developing a recommender system based on the information collected from this digital environment.

In order to generate recommendations, many traditional approaches have been already used in many applications and have excellent performances, such as content-based, collaborative filtering and hybrid approaches [14]. Others have also been proposed and studied recently, such as link prediction in graphs/networks [8] and topic modeling [9]. Among them, what attract our attention are context-aware approaches, which 
allows to generate more relevant recommendations through considering the specific contextual situations of the user [15]. Such situations are described by user context, which could be explicit or implicit [13]. To record user's contextual information, the user profile has been introduced and applied [19]. Therefore, we are interested in analyzing user contextual information and integrate it with user profile into user contextual profile to propose collaborator recommendations in collaborations.

The remainder of this paper is constructed as follows. Section 2 discusses user context and user profile, and studies context-aware recommender systems. Our user contextual profile is presented in Section 3. We apply a scenario to illustrate how to use our user contextual profile on recommending collaborators to a given user in the framework of collaborations in Section 4. We then discuss our collaborator recommendations and the proposed user contextual profile in Section 5. Finally, some conclusions and future work are put forward in Section 6.

\section{$2 \quad$ Related work}

In this section, we analyze two notions: user context and user profile. Moreover, we conduct a survey on context-aware recommender systems (CARSs).

\subsection{User context and user profile}

User context is a widely addressed concept in CARS since it matters in better predicting users' behaviors [17]. Usually, such contextual information can be collected from two approaches: requiring explicitly to the user and being learned implicitly from the user's behaviors [7]. However, user context remains dependent on applied domains and applications, such as user's intent [17], user's research topics [9]. According to the definition of context proposed by Dey [21], user context can be defined as any information that can be used to characterize the situation of a user who is considered relevant to the interaction with an application, including the user and applications themselves. Based on the properties of contextual information, it can be divided into static/dynamic context, long-term/short-term context [13].

As for user profile, its main features are user interests and preferences [16]. It depends on demographics or online user behaviors [7]. Based on the properties of different information in user profile, it is divided into two parts: static (e.g. user identity) and dynamic (e.g. current activities) [2]. While in [11], it is separated into short-term and long-term user profile depending on whether such information deals with users' current behaviors or not. In addition, the two main ways of collecting information for user profiles are also explicit and implicit [11, 16].

From these researches, it is obvious that user context and user profile have many features in common, such as collecting approaches, classifications. However, not any user contextual information could be included in user profile to express users' interests or preferences. For example, in a movie recommender system, user's companions [20] is critical contextual information that does not need to be recorded in the user's profile. In other words, user context is a larger concept than user profile. Conversely, 
user profile is included in user context. Since any information in user profile focuses only on users themselves, which definitely also belongs to user context.

Furthermore, a joint analysis of user context and user profile would provide a stronger comprehension of users. [17] uses a dataset of demographic, transactional and contextual information about the users to analyze users' behaviors. According to our discussions above, the first two parts belong to user profile, while the last one is under user context. Therefore, in our research, we are interested in integrating user context with user profile in order to construct user contextual profile, which could be used to predict users' behaviors in the framework of collaborations and thus generate corresponding recommendations.

\subsection{Context-aware recommender system}

Traditionally, recommender system (RS) deals with two types of entities: users and items [10]. Using a known set of users' ratings about items, these RSs are capable to predict those unknown ratings of users and thus recommend items based on the forecasted ratings. For these RSs, their rating function $R_{R S}$ is

$$
R_{R S}: \text { Users } \times \text { Items } \rightarrow \text { Ratings [15] }
$$

As for CARSs, they apply at least three types of information: users, items and context. Sometimes, it is even possible to construct a multi-dimensional CARS. In [10], context of a movie CARS is separated into 3 dimensions: Place, Time, and Companion. Thus, for an n-dimensional CARS, its rating function $R_{C A R S}$ is

$$
R_{C A R S}: D_{1} \times D_{2} \times \ldots \times D_{n} \rightarrow \text { Ratings [10] }
$$

where $D_{1}, D_{2}, \ldots, D_{n}$ represent its n dimensions (including Users, Items, ..).

To compare the different performances of CARS and RS, [17] demonstrates that knowing the context could help RS to perform better. Therefore, in order to generate more relevant recommendations, we are interested in constructing a CARS to recommend collaborators to a given user in the framework of collaborations. A similar research has been carried out in [9]: a first context-aware academic collaborator recommender system is built, where the context is referred to a set of topics that users will jointly work on. However, contextual information collected from users' historical collaborations is not utilized, such as users' historical interactions with others. Therefore, in our research, the context is referred to the contextual information obtained from users' historical collaborations in digital environments. We focus on utilizing such user context and integrating it into user contextual profile to predict unknown ratings of users on collaborators to generate collaborator recommendations in collaborations.

\section{User contextual profile in our CARS}

In this section, we introduce user contextual profile we built for collaborations in digital environments. Then the formalized elements of our CARS are presented. 


\subsection{User contextual profile}

We first introduce the relations between user profile and user context in collaborations. We discuss how to express users' interests and preferences properly and what contextual information could be collected from their historical collaborations. Finally, we propose a user contextual profile in a digital collaborative environment. In this profile, we retain demographic information and activity traces related to collaborations and discuss privacy protection of users' personal data.

\subsubsection{Relations between user profile and user context}

Based on the discussions in Section 2.1, user profile belongs to user context (as shown in Fig. 1). Besides, some of contextual information of users could be derived from their historical collaborations, as we discussed before in Section 2.2. In other words, user context is crossing with collaborations (as shown in Fig. 1).

Therefore, our user contextual profile is constructed based on user profile (represented by triangles) and user contextual information obtained from users' historical collaborations (represented by the intersected parts of circles and squares).

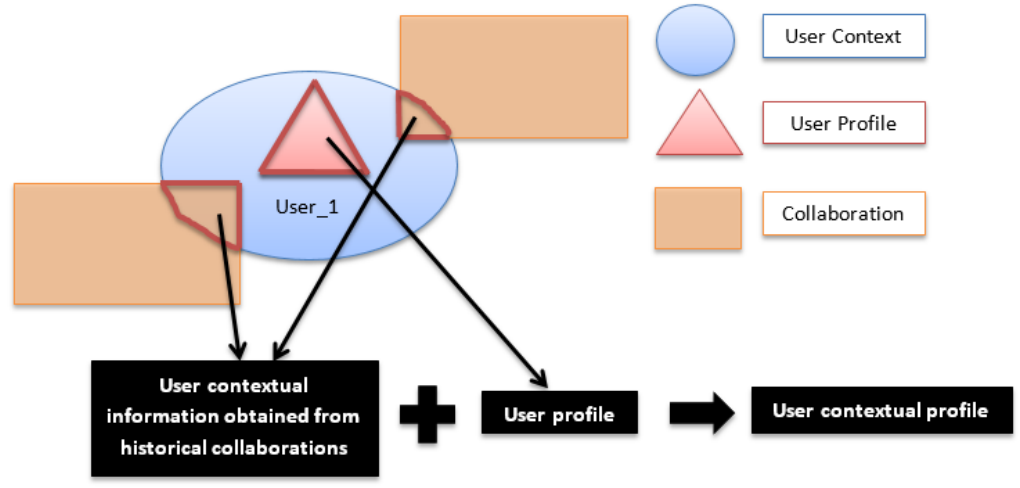

Fig. 1. User contextual profile in collaborations.

\subsubsection{User profile}

In our research, a user is a person who holds a user account in a digital environment that could provide digital support (such as tools and resources) to facilitate collaborations. According to [7], we apply demographic attributes to express user's personal characteristics in user profile (TABLE 1, part: Demographic attributes). Based on the values of these attributes, our CARS could predict users' possible collaborators. For example, by checking whether there is a common value of 'Spoken languages' between users, our CARS could determine whether they have adequate communication skills in collaborations. Besides, in order to identify users, we use 'Login' as a unique authentication.

\subsubsection{User context}

According to [1], a collaboration involves at least two persons and comprises a set of human actors' actions on behalf of the corresponding collaborator in order to achieve 
a shared goal. Thus, in a digital environment, collaboration necessities at least two members and a shared goal among all the members. To represent properly collaborations in digital environments, we use 'user group'. A user group consists of at least two users with a shared objective. These users could communicate and interact with each other in the user group to advance their collaboration. Moreover, digital environments facilitate to implicitly gather contextual information. User activity traces could be easily recorded [18], which are associated with the user and the corresponding user group.

Therefore, in a digital collaborative environment, users are related to user groups, their collaborators and their activity traces, which are their contextual information derived from their historical collaborations and will be used to calculate the contextual attributes in user contextual profile (TABLE 1, part: Contextual attributes).

\subsubsection{User contextual profile in the framework of collaboration}

Based on the discussion above, our contextual profile contains two parts (shown in TABLE 1): demographics attributes, and contextual attributes.

TABLE 1. User contextual profile.

\begin{tabular}{l|l}
\hline & \multicolumn{1}{c}{ Attributes } \\
\hline $\begin{array}{l}\text { Demographic } \\
\text { attributes }\end{array}$ & $\begin{array}{l}\text { Login; Gender; Competencies; Age; Educational level; } \\
\text { Spoken languages; Employment; Home Country; Home City }\end{array}$ \\
\hline $\begin{array}{l}\text { Contextual } \\
\text { attributes }\end{array}$ & Historical collaborations; Diversity of collaborators; Activeness \\
\hline
\end{tabular}

In particular, the contextual profile require collecting users' personal information and activity traces, which raises our concerns about users' personal data ${ }^{1}$ privacy protection. Europe has enforced General Data Protection Regulation (GDPR) since 25 May $2018^{2}$. Thus, following GDPR, we ask users for their consents to collect and process their personal data in our CARS. Under the authorizations of users, they allow us to use their personal information and activity traces gathered through their historical collaborations to generate collaborator recommendations. All the collected data is only kept for users themselves to improve their collaborations and will not be shared with any third party.

\subsection{Formalized elements}

This section presents the formalized terminologies that are used to formulate our collaborator recommendation problem. Then the definition and calculation of contextual attributes in user contextual profile are also explained.

1 According to [22], personal data in GDPR indicates information that can identify an individual directly or indirectly, specifically including online identifiers (e.g., IP addresses, cookies and digital fingerprinting, and location data).

2 https://eur-lex.europa.eu/legal-content/EN/TXT/PDF/?uri=CELEX:32016R0679\&from=EN 


\subsubsection{Terminology}

For a user $u$, who is in a user group $g(u \in g)$ at time $t$, the clarifications of terminologies are: 1) Collaborator: a collaborator $c$ of $u$ at time $t$ is another user $c(\neq u)$ who is in the same user group $g(c \in g)$ at time $t$; 2) Contact (possible collaborator): a contact $c_{p}$ of $u$ at time $t$ is another user $c_{p}(\neq u)$ who is not in the user group $g\left(c_{p} \in\right.$ $g)$ at time $t$.

Considering that collaborators and contacts are definitely users, we consider profile and context of collaborators and contacts are equal to user profile and user context. Besides, we use CCU to represent Contact/Collaborator/User in the following: 1) CCU profile consists of CCU's demographic information; 2) CCU context includes all the information that can be used to characterize the situation of a CCU. For the time being, in our research, CCU context is limited to the contextual information that is obtained from historical collaborations and CCU profile (shown in Fig. 1); 3) CCU contextual profile merges the information in CCU profile and CCU context derived from historical collaborations, including demographic attributes and contextual attributes (shown in TABLE 1).

Thus, our context-aware collaborator recommendation problem is formulated as: Given a user $u$ and his/her current user groups ${ }^{3} \boldsymbol{G}=\left\{g_{1}, g_{2}, \ldots, g_{m_{u}}\right\}$, K (specified by user $u$ ) contacts (possible collaborators) $c_{p}$ from all candidates $R_{p}=\left\{c_{p} \notin\right.$ $\left.g_{i}, c_{p} \neq u\right\}\left(i \in\left\{1,2, \ldots, m_{u}\right\}\right)$, who will collaborate with $u$ with the highest probabilities. $\boldsymbol{m}_{\boldsymbol{u}}$ represents the number of current user groups that user $u$ is in. Besides, in our CARS, the recommended items to a given user are his/her contacts (possible collaborators). Then the rating function $R_{c o l}$ of our CARS is:

$$
R_{c o l} \text { : User contextual profile } \times \text { Contact contextual profiles } \rightarrow \text { Ratings }
$$

\subsubsection{Contextual attributes}

The three contextual attributes are defined and calculated using contextual information derived from users' historical collaborations.

For the first attribute 'historical collaborations', its values are a list of all historical user groups in which the user has participated. Here, historical user groups mean that the represented collaborations are already terminated, no longer in progress.

Then, the diversity of collaborators of a user $u$ is measured by the entropy of historical collaborators ${ }^{4}$ (denoted by $H_{u}$ ). According to [9], a large $H_{u}$ indicates a user prefers to collaborate with different collaborators. In our research, $H_{u}$ represents a logarithmic measure of the number of historical collaborators with a significant probability of working together, which follows:

$$
H_{u}=-\sum_{u^{\prime}} p_{u^{\prime}} \ln p_{u^{\prime}}\left(\sum_{u^{\prime}} p_{u^{\prime}}=1, u^{\prime} \neq u\right)
$$

3 Current user groups mean that represented collaborations have not finished yet, still in progress.

4 Historical collaborators represent the contacts that user $u$ has collaborated with in his/her historical collaborations. 
where $p_{u^{\prime}}=\frac{N_{u, u \prime}}{N_{u}}$ represents the probability of collaborating with $u^{\prime}$ according to user $u$ 's historical collaborations records; $N_{u, u}$ is the number of collaborations that user $u$ collaborated with user $u^{\prime} ; N_{u}$ indicates the number of collaborations that user $u$ collaborated with others in all historical collaborations; the interval of $H_{u}$ is $\left(0, \ln N_{u}\right]$. Finally, the activeness of a user in collaborations aims at analyzing the willingness of the user to take actions and contribute to the collaboration itself. The activeness is measured by the average activity rate of the user $u$ in historical user groups (denoted by $\left.\overline{A_{u}}\right)$ :

$$
\overline{A_{u}}=\frac{1}{n_{u}} \sum_{j=1}^{n_{u}} A_{u, g_{j}}
$$

where $A_{u, g_{j}}$ is the number of activities that user $u$ effected in the historical group $g_{j}$; $n_{u}$ indicates the number of historical user groups that user $u$ was in; the interval of $\overline{A_{u}}$ is $[0,+\infty)$.

\section{Using user contextual profile for collaborator recommendations}

In this section, we illustrate a scenario of Emma to discuss how to predict users' preferences on collaborators through user contextual profile: Emma is a Ph.D. student in $X$ laboratory. Her thesis is about data mining and user modeling, supervised by Elsa and Marie. During her thesis, Emma has collaborated with several people on three projects. Moreover, there are two other persons in X laboratory: Marinela and Jack. However, both of them never collaborate with Emma before. Now, Emma is looking for several partners to develop an application in order to analyze users' behaviors in her laboratory. However, she doesn't know who she will collaborate. To help Emma, our CARS uses her contextual profile to predict a ranked list of all possible collaborators and recommend the top 3 ( $\mathrm{K}=3$, specified by herself) collaborators to her. She can choose appropriate ones from the recommended collaborators to collaborate with.

TABLE 2. Emma's contextual profile.

\begin{tabular}{l|c}
\hline \multicolumn{1}{c|}{ Attributes } & Example of Emma \\
\hline Login & Emma_account \\
\hline Gender & Female \\
\hline Competencies & User modeling, Data mining \\
\hline Age & 25 \\
\hline Educational level & Ph.D. student \\
\hline Spoken languages & French, English, Chinese \\
\hline Employment & X laboratory \\
\hline Home Country & France \\
\hline Home City & Compiègne \\
\hline
\end{tabular}




\begin{tabular}{l|c}
\hline Historical collaborations (IDs) & $g_{1}, g_{2}, g_{3}$ \\
\hline Diversity of collaborators $H_{u}\left(H_{u} \in\left(0, \ln N_{u}\right]\right)$ & 1.332 \\
\hline Activeness $\overline{A_{u}}\left(\overline{A_{u}} \in[0,+\infty)\right)$ & 6 \\
\hline
\end{tabular}

In our scenario, suppose Emma's historical collaborations are represented by three user groups: $g_{1}, g_{2}$ and $g_{3}$. According to the recorded traces in the groups, she has acted 7 times in $g_{1}, 6$ times in $g_{2}$ and 5 times in $g_{3}$.

Then, Emma gets $g_{1}, g_{2}, g_{3}$ for the first contextual attribute. Besides, Emma has collaborated 2 times with Marie (in $g_{1}, g_{2}$ ), 1 time with Oriane (in $g_{1}$ ), 1 time with Nathalie (in $g_{2}$ ), and 1 time with John (in $g_{3}$ ), then we have $N_{E m m a}=2+1+1+$ $1=5 \quad$ and $\quad H_{E m m a}=\left(\frac{2}{5} \ln \frac{2}{5}+\frac{1}{5} \ln \frac{1}{5}+\frac{1}{5} \ln \frac{1}{5}+\frac{1}{5} \ln \frac{1}{5}\right)=1.332 \quad$. Considering $\ln N_{E m m a}=\ln 5=1.609$, Emma gets a large $H_{E m m a}(0 \ll 1.332<1.609)$. As for the third one, Emma has acted separately 7 times (in $g_{1}$ ), 6 times (in $g_{2}$ ) and 5 times (in $g_{3}$ ), then her activeness is $\overline{A_{E m m a}}=\frac{7+6+5}{3}=6$, which means that Emma performs 6 activities in a single collaboration on average. Finally, Emma's contextual profile is shown in TABLE 2.

Once Emma's contextual profile is complete, we need her contacts' contextual profiles. All her contacts work in X laboratory. And suppose that from their contextual profiles, we can know the following information (presented in TABLE 3).

TABLE 3. Information from Emma's contacts' contextual profiles.

\begin{tabular}{l|l}
\hline Contact & Information \\
\hline Jack & Speaks French and English; $\overline{A_{\text {Jack }}}=10, H_{\text {Jack }}=1.040$. \\
\hline Marinela & Speaks English; $\overline{A_{\text {Marrnela }}}=1, H_{\text {Marinela }}=0.040$. \\
\hline John & $\begin{array}{l}\text { Speaks French, English and Spanish; Has collaborated with Emma } \\
\text { once in } g_{3} ; \overline{A_{\text {John }}}=5, H_{\text {John }}=1.002 .\end{array}$ \\
\hline
\end{tabular}

Based on TABLE 3, all Emma's contacts are possible to collaborate with her since they all work in a same place and speak at least one common language. However, according to Emma's contextual profile (shown in TABLE 2), she gets a large $H_{\text {Emma }}$ which indicates that when compared to contacts that she has already collaborated with, Emma prefers to work with other contacts. Thus, comparing with John, Emma would prefer to work together with Jack or Marinela. Besides, Emma is an active collaborator since she is willing to take actions in collaborations, averagely 6 times in a single one. However, Marinela is not an active collaborator based on the value of $\overline{A_{\text {Marınela }}}$. Then, Emma would rather not collaborate with Marinela. Therefore, Emma's top 3 possible collaborators is $R_{\text {col }}(\mathrm{Jack})>R_{\text {col }}(\mathrm{John})>$ $R_{\text {col }}$ (Marinela).

In our scenario, user contextual profile contributes to generating collaborator recommendations. On the one hand, Emma's contextual profile concentrates on her personal preferences in collaborations. On the other hand, her contacts' contextual profiles (Jack, John, Marinela) help our CARS to calculate Emma's possible ratings for them. 


\section{Discussion}

The generated recommendations are directly related to the user contextual profile. More information we have in the user contextual profile, more accurate the recommendation will be. We are therefore facing to two critical issues: how to improve the record of users' activity traces from a digital collaborative environment; how to let users agree to share their personal information and activity traces in the environment according to GDPR.

Using user contextual profile to generate recommendations offers diverse possibilities at research and practice level in future. The proposed user contextual profile could be broadened by widening the scope of collecting information in digital environments, such as specifying details of activity traces. Currently, we only apply the number of activities that user effected in historical collaborations, without any detailed information of an activity. Thus, we could enrich the user contextual profile by specifying the details of an activity in a user group, such as: one or multiple actor(s), time, resources used, and type of activity. Such profile could also be filled by the information coming from different software that users used for their collaborations. In addition, users need to give their agreements to apply this information to generate collaborator recommendations.

\section{Conclusion and future work}

In this paper, we focus on recommending possible collaborators to a given user in the framework of collaborations and propose a user contextual profile to generate recommendations. Based on the related literature in Section 2, we have explained why user contextual profile is needed to provide collaborator recommendations and justified the choice of building a CARS. We then presented the user contextual profile built for collaborations in digital environments and demonstrated how to use it in our CARS by illustrating a scenario. We finally discuss the issues of our recommendations and explore possibilities of the proposed user contextual profile in future.

Our research perspectives include the exploration of the relations between user context and collaboration context, as well as the implementation of the recommendation algorithms for collaborations in digital environments.

\section{References}

1. Li, S., Abel, M. H., Negre, E.: Contact and Collaboration Context Model. In: 2018 IEEE $4^{\text {th }}$ International Forum on Research and Technology for Society and Industry. IEEE (2018).

2. Tazari, M. R., Grimm, M., Finke, M.: Modeling user context. In: Proceedings of the $10^{\text {th }}$ International Conference on Human-Computer Interaction, Crete (2003).

3. Mattessich, P.W., Monsey, B.R.: Collaboration: what makes it work. A review of research literature on factors influencing successful collaboration. Amherst H. Wilder Foundation, 919 Lafond, St. Paul, MN 55104. (1992). 
4. Patel, H., Pettitt, M., Wilson, J.R.: Factors of collaborative working: A framework for a collaboration model. In: Applied ergonomics, vol. 43(1), pp. 1-26 (2012).

5. Godwin-Jones, R.: Blogs and wikis: Environments for online collaboration. (2003).

6. Boley, H, Chang, E.: Digital ecosystems: Principles and semantics. In: Digital EcoSystems and Technologies Conference, pp 398-403 (2007).

7. Anand, S.S., Mobasher, B.: Introduction to intelligent techniques for web personalization. ACM Transactions on Internet Technology (TOIT), 7(4), 18 (2007).

8. Li, J., Xia, F., Wang, W., Chen, Z., Asabere, N.Y., Jiang, H.: Acrec: a co-authorship based random walk model for academic collaboration recommendation. In: Proceedings of the $23^{\text {rd }}$ International Conference on World Wide Web, pp. 1209-1214. ACM (2014).

9. Liu, Z., Xie, X., Chen, L.: Context-aware Academic Collaborator Recommendation. In: Proceedings of the 24th ACM SIGKDD International Conference on Knowledge Discovery \& Data Mining, pp. 1870-1879. ACM (2018).

10. Adomavicius, G., Sankaranarayanan, R., Sen, S., Tuzhilin, A.: Incorporating contextual information in recommender systems using a multidimensional approach. ACM Transactions on Information Systems (TOIS) 23(1), 103-145 (2005).

11. Shen, X., Tan, B., Zhai, C.: Implicit user modeling for personalized search. In: Proceedings of the 14th ACM international conference on Information and knowledge management, pp. 824-831. ACM (2005).

12. Yang, C., Liu, T., Liu, L., Chen, X.: A Nearest Neighbor Based Personal Rank Algorithm for Collaborator Recommendation. In: 2018 15th International Conference on Service Systems and Service Management (ICSSSM), pp. 1-5. IEEE (2018).

13. Shen, X., Tan, B., Zhai, C.: Context-sensitive information retrieval using implicit feedback. In: Proceedings of the 28th annual international ACM SIGIR conference on Research and development in information retrieval, pp. 43-50. ACM (2005).

14. Resnick, P., Varian, H.R.: Recommender systems. Communications of the ACM 40(3), 56- 58 (1997)

15. Adomavicius, G., Tuzhilin, A.: Context-aware recommender systems. In: Recommender systems handbook, pp. 217-253. Springer, Boston, MA (2011).

16. Tamine-Lechani, L., Boughanem, M., Daoud, M.: Evaluation of contextual information retrieval effectiveness: overview of issues and research. Knowledge and Information Systems, 24(1), 1-34 (2010).

17. Palmisano, C., Tuzhilin, A., Gorgoglione, M.: Using context to improve predictive modeling of customers in personalization applications. IEEE transactions on knowledge and data engineering, 20(11), 1535-1549 (2008).

18. Wang, N., Abel, M. H., Barthès, J. P., Negre, E.: Recommending competent person in a digital ecosystem. In: Industrial Informatics and Computer Systems (CIICS), 2016 International Conference, pp. 1-6. IEEE (2016).

19. Golemati, M., Katifori, A., Vassilakis, C., Lepouras, G., Halatsis, C.: Creating an ontology for the user profile: Method and applications. In: Proceedings of the first RCIS conference, pp. 407-412 (2007).

20. Ostuni, V.C., Di Noia, T., Mirizzi, R., Romito, D., Di Sciascio, E.: Cinemappy: a Contextaware Mobile App for Movie Recommendations boosted by DBpedia. SeRSy, 919, 37-48 (2012).

21. Dey, A.K.: Understanding and using context. Personal and ubiquitous computing, 5(1), 4-7 (2001).

22. Goddard, M.: The EU General Data Protection Regulation (GDPR): European regulation that has a global impact. International Journal of Market Research, 59(6), 703-705 (2017). 